\title{
First dorsal metacarpal artery flap for thumb reconstruction: a retrospective clinical study
}

\author{
Thomas Muyldermans · Robert Hierner
}

Received: 20 November 2008/Accepted: 9 March 2009/Published online: 2 April 2009

(C) Springer-Verlag 2009

\begin{abstract}
Extensive pulp (zone 4) defects of the thumb, with the exposure of tendon or bone, are challenging reconstructive problems. Surgical treatment includes the use of local, regional, and free flaps. The first dorsal metacarpal artery flap has been used successfully for defects of the thumb. The innerved first dorsal metacarpal artery flap from the dorsum of the index finger was first described by Hilgenfeldt and refined by Holevich. An island flap carried on a neurovascular pedicle consisting of the first dorsal metacarpal artery was first demonstrated by Foucher and Braun. Seven innervated FDMCA island flaps were performed from May 2005 until July 2007 for thumb reconstruction. There were three women and four men with an average age of 54.9 years (range 28-89 years). The mean follow-up period was 15.4 months (range 4-29 months). The dominant hand was involved in six $(85.7 \%)$ patients. In a retrospective clinical study, the following criteria were evaluated: (1) etiology of the defect, (2) time of reconstruction (primary vs. delayed), (3) survival rate of flap, (4) sensory function (Semmes-Weinstein monofilaments, static 2-PD, pain, cortical reorientation), (5) TAM measured with the Kapandji index, and (6) subjective patient satisfaction (SF 36). Four patients presented with trauma, two patients with defects after tumor resection and one with infection of the thumb. The flap was used for immediate reconstruction in three $(42.9 \%)$ patients and for delayed reconstruction in four $(57.1 \%)$ patients. Delayed reconstruction was performed 4.75 (1-12) months after
\end{abstract}

T. Muyldermans $\cdot$ R. Hierner $(\bowtie)$

Department of Plastic, Reconstructive and Aesthetic Surgery,

Centre for Interdisciplinary Reconstructive Surgery,

Microsurgery, Hand Surgery, Burns, University Hospital

Gasthuisberg, Catholic University of Leuven, Herestraat 49,

3000 Leuven, Belgium

e-mail: robert.hierner@uk-essen.de initial trauma or first surgery. The donor area was grafted with full-thickness skin grafts in all cases. All flaps survived. The mean SWMF was $3.31 \mathrm{~g}$ and average statis 2-PD over the flap was $10.57 \mathrm{~mm}$. Pain at the flap scored 3.71 over 10 and at the donor site 2.17 over 10. Paresthesia at the flap scored 0.57 over 4 and at the donor site 0.33 over 4 . Complete cortical reorientation was only seen in one patient. The mean Kapandji score of the reconstructed thumb was 7.43 over 10 . Using the SF-36, mean physical health of the patients scored $66.88 \%$ and mean mental health scored $70.55 \%$. Disturbing pain and paresthesia of the flap are exceptional. The static 2-PD is more than $10 \mathrm{~mm}$, and is clinically over the limit. Cortical reorientation was incomplete in all but one patient. Touch on thumb is felt on the dorsum of the index finger; however, sensation is not disturbing or interfering with the patient's activities. Foucher described the technique débranchement-rébranchement in order to improve this problem. The postoperative total amount of motion of the reconstructed thumb was very good. The results demonstrated that the FDMCA flap has a constant anatomy and easy dissection. It has a low donor site morbidity if FTSG is used. It also shows good functional and aesthetic results. Therefore, the FDMCA flap is a first treatment of choice for defects of the proximal phalanx and proximal part of the distal phalanx of the thumb.

Keywords Hand - Trauma - Thumb - Flap . First dorsal metacarpal artery $\cdot$ Reconstruction

\section{Introduction}

Extensive pulp (zone 4) defects of the thumb, with the exposure of tendon or bone, are challenging reconstructive problems. Surgical treatment includes the use of local, 
regional, and free flaps. The first dorsal metacarpal artery flap has been used successfully for dorsal defects of the thumb. The innerved first dorsal metacarpal artery flap from the dorsum of the index finger was first described by Hilgenfeldt [1] and refined by Holevich [2]. An island flap carried on a neurovascular pedicle consisting of the first dorsal metacarpal artery was first demonstrated by Foucher and Braun [3]. Gebhart and Meissl [4] described an extended first dorsal metacarpal artery neurovascular island flap.

\section{Surgical anatomy}

The first dorsal metacarpal artery (FDMCA) arises from the radial artery in the first intermetacarpal space, just distal to the tendon of the extensor pollicis longus. The artery divides into the radial branch to the thumb, the intermediate branch to the first web space, and the ulnar branch to the index finger. In $90 \%$ of cases, the FDMCA parallels the second metacarpal bone distally. In $10 \%$ of cases, it is found in the midline of the triangle that is formed by the first commissura. The FDMCA is in a superfascial $(57 \%)$ or subfascial $(43 \%)$ location.

On its way distally, the artery sends cutaneous, muscular, and osteal branches. The cutaneous branches carefully supply the skin of the proximal interphalangeal joint by an extended fascial-cutaneous vascular network to the base of the second metacarpal bone. The muscular branches supply the interosseus dorsalis I muscle. The osteal branches provide the second metacarpal bone. So, in practice, cutaneous, muscular and osteal flaps are possible. In this article, we describe the cutaneous FDMCA flap.

Functionally important anastomoses exist through several branches to the second dorsal metacarpal artery and to the palmar vascular system. The latter anatomosis is formed by a branch that originates from the FDMCA at the level of the head of the second metacarpal bone or near the metacarpophalangeal joint. Moreover, several variants are described. Nevertheless this doesn't influence the operative handling.

In addition to the FDMCA, in $27-86 \%$ of cases there is a superficial artery that runs together with the superficial branch of the radial nerve, parallel to the FDMCA in the superficial layer of the subcutaneous adipose tissue. It anastomoses distally with the FDMCA. Because of its course, this vessel is also considered as the superficial FDMCA. If both arteries are present, they form an extended epi- and subfascial vascular system. One or two cutaneous veins run, superficially, to the extensor tendon, towards the subcutaneous adipose tissue of the first intermetacarpal space [5]. Additionally, two small comitant veins, which are in connection with the large superficial cutaneous veins, accompany the FDMCA.

On the dorsal aspect of the hand, the superficial branch of the radial nerve divides into four or five dorsal digital nerves. These nerves provide branches to the skin of the radial dorsum of the hand and innervate the dorsum of the thumb, index and middle phalanx of the third finger. The superficial branch of the radial nerve anastomoses with the dorsal branch of the ulnar nerve. Multiple variants of this anastomosis are described [5].

\section{Operative technique}

The patient is placed in a supine position, and the arm is positioned on the arm table. The operation is done in plexus block or under general anesthesia. The upper limb and the ipsilateral groin are prepared and draped. A tourniquet (250$300 \mathrm{mmHg}$ ) is applied at the upper arm. After debridement of the thumb defect, the skin flap is outlined. The flap is harvested from the dorsal aspect of the index finger, including the first dorsal metacarpal artery and a branch of the superficial radial nerve as a pedicled flap. The maximum size of the skin island includes the dorsal skin of both the proximal and middle phalages along the midradial and midulnar lines of the index finger. To reduce the donor site mobidity, the dorsal skin of the metacarpophalangeal joint and proximal joint should be preserved. Between the bases of the first and second metacarpal bone, the tip of the triangular first web space can be palpated, indicating the most proximal point of the pedicle dissection. When marked, this point represents the pivot point of the flap. Because it is recommended to include a large subcutaneous vein in the pedicle, the skin incision is continued along the radial aspect of the second metacarpal bone. Elevation of the skin island commences distally and radially, preserving the paratenon over the extensor apparatus. Dissection of the pedicle should be carried towards the site of origin of the FDMCA.

A critical point in the dissection is the radial aspect of the extensor hood of the metacarpophalangeal joint. Although the ulnar branch of the FDMCA is tiny and courses deeply within the musculo-osseus groove, no attempt is made to visualise the artery. Instead, safe dissection can be achieved by including the radial shaft periosteum of the second metacarpal bone, continued by the ulnar head fascia of the first dorsal interosseus muscle. Any small arterial branches penetrating the aponeurosis of the first dorsal interosseus muscle must be carefully ligated or coagulated. By doing so, the maximum potential length of the flap pedicle is achieved. The pedicle includes the fascia of the first dorsal interosseus muscle, the dorsal veins, and the sensory branch of the radial nerve. After raising the flap, the tourniquet is released and vascular flow of the flap is ascertained. The flap is then transferred into the pulp defect of the thumb.

With the Hilgenfeldt technique [1], the skin remains intact towards the base of the flap. 
With the Holevich technique [2], the skin island over the pedicle is smaller and gives the flap the form of a tennis racquet or a flag. This avoids the excess of skin in the first commisure region (Fig. 1a-g).

With the Foucher technique [3], there is no skin over the neurovascular pedicle. In this case, a subcutaneous tunnel is made along the ulnar aspect of the thumb from the snuffbox to the proximal margin of the pulp defect to tranfer the flap into the defect by gentle traction.

The donor site is grafted with a full-thickness skin graft.

\section{Patients and methods}

Seven innervated FDMCA island flaps were performed from May 2005 until July 2007 for thumb reconstruction. There were three women and four men with an average age of 54.9 years (range 28-89 years). The mean follow-up period was 15.4 months (range 4-29 months). The dominant hand was involved in six $(85.7 \%)$ patients. All flaps survived. The donor area was grafted with full-thickness skin grafts in all cases.
Fig. 1 Reconstruction of a complete palmar distal phalanx defect (zone 4 defect) of the left hand in a manual worker caused by a circle saw. a Clinical aspect at admission to the hospital; b intraoperative aspect: planning the flap; c intraoperative aspect: raising the flap; d postoperative aspect: the donor site is grafted with a fullthickness skin graft, fixed by a tie-over dressing; e clinical aspect after 1 year: dorsal view (there is no restriction within the first web space); $\mathbf{f}$ clinical aspect after 1 year: palmar view (there is no restriction within the first web space); $\mathbf{g}$ clinical aspect after 1 year: Kapandji index $9 / 10$
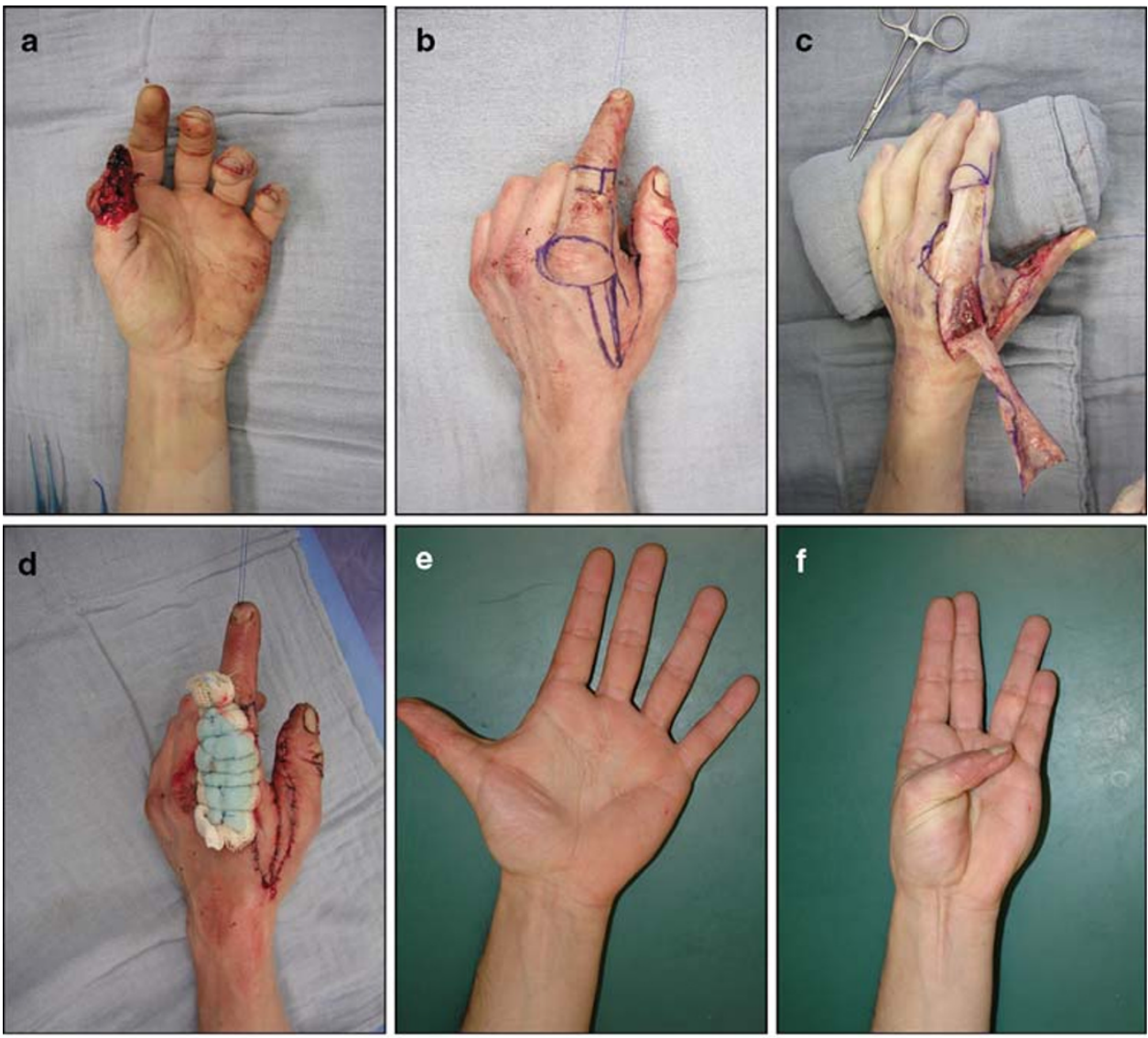

g

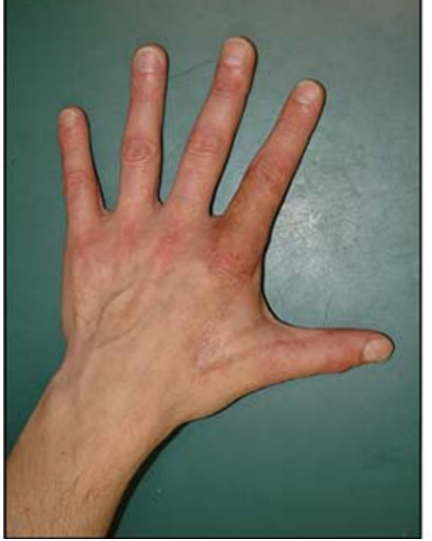


Sensory function was evaluated with Semmes-Weinstein monofilaments (SWMF) and static 2-point discrimination (s2-PD) testing.

An analogue scale was used to subjectively evaluate paresthesia $(0=$ no paresthesia, $4=$ severe paresthesia), pain $(0=$ no pain, $10=$ maximal imaginable pain $)$ and cosmetics $(0=$ worst cosmetic result, $10=$ best cosmetic result) of both the flap and donor site. Cortical reorientation was tested with a needle prick, asking the patient whether the stimulus seemed to be coming from the thumb or from the donor finger. The mobility of the first ray was evaluated with the Kapandji score.

Patients were asked about restrictions in activities of daily living (ADL) and their global satisfaction with the result, using the SF-36. The 36 questions deal with eight categories about both physical and mental health on a scale from 0 to $100 \quad(0=$ worst result, $100=$ best result $)$ (Table 1).

\section{Results}

The results of all patients are shown in Table 1. Four patients presented with trauma, two patients with defects after tumor resection, and one patient with infection of the thumb. The flap was used for immediate reconstruction in three $(42.9 \%)$ patients and for delayed reconstruction in four $(57.1 \%)$ patients. Delayed reconstruction was performed at 4.75 months [1-12] after initial trauma or first surgery. The donor area was grafted with full-thickness skin grafts in all cases. All flaps survived.

The results of the sensory function of the flap area are an average Semmes-Weinstein score of $3.31 \mathrm{~g}$ and an average static two-point discrimination of $10.57 \mathrm{~mm}$.

The pain scale reports an average of 3.71 over 10 at the flap area and an average of 2.17 over 10 at the donor site. The average score of possible paresthesia at the flap area is 0.57 over 4 and at the donor site is 0.33 over 4 . The donor site in case 4 could not be evaluated because in that case digit 2 was amputated. Cortical reorientation was complete in one patient. The average Kapandji score in this study was 7.43 over 10 . Using the SF-36, mean physical health of the patients scored $66.88 \%$ and mean mental health scored $70.55 \%$.

\section{Discussion}

The FDMCA island flap is harvested from the dorsal aspect of the index finger including the first dorsal metacarpal artery with its concomitant veins and a branch of the superficial radial nerve as a pedicled neurovascular flap. It was first described by Hilgenfeldt [1], refined by Holevich [2], and modified as a pure island flap by Foucher and Braun [3]. It has a wide arch of rotation and easily reaches the palmar or radial aspects and the pulp of the thumb. The venous drainage of the concomitant veins of the FDMCA is very reliable, the possible size of the flap is big, and the donor site morbidity is low.

Extensive pulp defects of the thumb, with the exposure of tendon or bone, are challenging reconstructive problems because of the lack of locally available tissue. The main goals of thumb reconstruction are the preservation of length and sensibility. Surgical treatment includes the use of local, regional, and free flaps. In 1953, Littler [6] described a neurovascular island flap, harvested from the ulnar aspect of the ring or middle finger. Various microsurgical free flaps from the first and second toe and web space of the foot were described as alternative solutions in 1978 and 1979 [7-9].

The FDMCA flap is indicated in patients with defects of the proximal and distal phalanx zone 4. Indications for the Hilgenfeldt technique are palmar or dorsal defects of the proximal and distal phalanx. Holevich and Foucher techniques are indicated for palmar or dorsal zone 4 defects of the distal phalanx. Other possibilities of reconstruction of zone 4 defects are the Littler flap, the cross-finger flap, and the partial toe transfer. In the case of partial toe transfer, the patient has to be young, motivated, and very compliant. Zone 2 and 3 defects are covered with a Moberg flap or one of its modifications [5]. Defects of the proximal phalanx and the first web space can be reconstructed with the posterior interosseus flap and the radialis forearm flap, depending on the size of the defect.

Contraindications of the FDMCA flap are circular defects at the proximal or distal phalanx and previous injury at the second metacarpal level.

Ratcliffe et al. [10] described the use of the FDMCA flap to cover extensive pulp defects in the normal-length thumb. In the five patients they reported, there was no morbidity related to the donor area on the dorsum of the index finger, and flap survival was $100 \%$. Local flap coverage is particularly difficult when thumb length is preserved, but pulp loss is extensive, exposing structures in the distal thumb. Foucher and Braun described the FDMCA flap to resurface dorsal thumb defects or the stumps of shortened thumbs, but they did not comment on its use for pulp loss in the normal length thumb.

Sherif [11, 12] reported a study of 23 patients with FDMCA flaps, 18 fasciocutaneous flaps, and 5 fascial flaps. Anatomical indications of proximal-based FDMCA fasciocutaneous flaps were first web space reconstruction (congenital, postburn, or posttraumatic), thumb palmar surface (avulsion or electric burn), thumb dorsal surface 


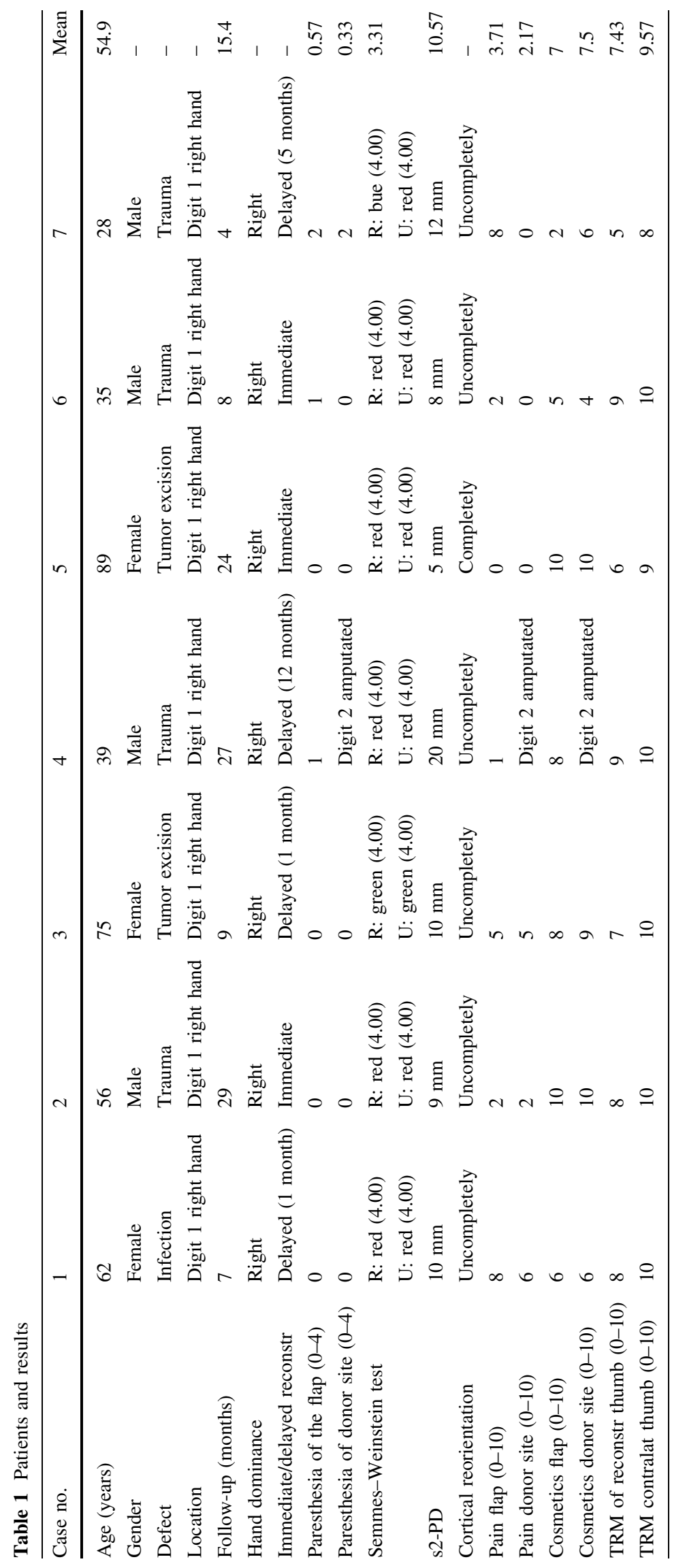


(electric burn), and dorsal surface of the hand (bomb blast injury). In the 18 patients with fasciocutaneous flaps, there was no flap necrosis. The skin grafts, applied to the donor area, were satisfactory.

Eski et al. [13] described the use of the FDMCA flap in thumb deformities after burns. They stated that the FDMCA flap can provide elastic, durable, and sensate coverage for soft tissue defects after contracture release. In a 3-year period, the neurovascular island FDMCA flap was used in 14 patients suffering thumb deformities. Follow-up revealed that all deformities were successfully treated with satisfactory functional recovery and cosmetic results. Donor site morbidity was minimal with an acceptable scar on the dorsum of the index finger and adequate tendon gliding without producing an extension deficit.

Pellisier et al. [14] reported a review of 27 cases with reverse dorsal digital and metacarpal flaps. Reverse dorsal digital and metacarpal flaps use the dorsal skin of the digital or metacarpal areas, and they are based on the arterial branches anastomosing the volar and dorsal arterial networks of the fingers. These flaps are transposed as reverse island flaps. These flaps, however, do not seem suitable for restoring sensitivity. A series of 27 flaps was reviewed, with more than 6 months of follow-up. Skin defects in all patients were located over or beyond the proximal interphalangeal joint as far as the fingertip and were combined with bone, joint, or tendon exposure. No flap necrosis was observed, and donor site morbidity was minimal. Reverse digital dorsal and metacarpal flaps combine the advantages of an extended skin paddle and a versatile pivot point on the phalanx and allow coverage of wide and distal defects. Although the first dorsal metacarpal artery supplies only the proximal portion of the index finger, harvesting the dorsal skin over the middle phalanx as a random extension is possible because of the existence of the rich dermal-subdermal plexus supplying the dorsal skin of both segments.

El-Khatib [15] reported his clinical experiences with the extended first dorsal metacarpal artery island flap for thumb reconstruction. This surgical procedure was created to provide composite resurfacing of total palmar or dorsal defects of a thumb with normal length. The procedure involved a flap that included the dorsal skin from both the proximal and middle phalanges of the index finger. Harvesting the dorsal skin of both the proximal and middle phalanges of the index is considered a modified version of the FDMCA flap. According to the author, the extension is possible because of the existence of the rich dermal-subdermal plexus supplying the dorsal skin. His other argument is that all axial pattern skin flaps can have an arterial and a cutaneous random pattern portion, the socalled "dynamic territory," which depends on adequate perfusion pressure. El-Khatib described five cases. Flap survival was excellent, and grafting at the donor site was successful in all cases.

Gebhart and Meissl [4] reported the successful use of an extended island FDMCA wraparound flap for thumb reconstruction in one case.

Important concerns about pedicled neurovascular island flaps include the dual-location phenomenon and the problems of cortical reorientation.

Cortical reorientation is the fact that the brain recognizes a stimulus from the flap area as a stimulus from the thumb, and not from the index finger. This process takes some time, but is usually complete after 2 years. We saw a complete cortical reorientation of the Foucher flap with only one patient. The incomplete reorientation with six of our patients was reported not to be disturbing and did not interfere with their daily activities. If, however, the patient wishes, this can be corrected surgically with a technique described by Foucher [3], "débranchement-rébranchement." The divided nerve of the transferred island flap is sutured to the original nerve of the thumb.

As we studied the cortical reorientation of the FDMCA flap, Yoshinori [16] studied the cortical reorientation of the Littler flap. He conducted a study to examine the sensory function of thumbs that were reconstructed using two procedures: the original Littler neurovascular island flap (NVIF) procedure and a modification of the NVIF procedure in which the divided nerve of the transferred island flap is sutured to the original nerve of the thumb. This is the procedure of "débranchement-rébranchement" according to Foucher. Twenty-one patients with a mean follow-up period of 4.6 years were examined. Nine patients were treated with the NVIF procedure and 12 with the modified NVIF procedure. Paresthesia was observed more frequently in the patients treated with the original NVIF procedure. The Semmes-Weinstein test and the static and moving 2-point discrimination examinations showed no significant difference between the two groups. The transferred pedicle flap was fully recognized as representing the thumb in the modified procedure. In the original procedure, however, the sensibility of the transferred pedicle flap was recognized as coming from the thumb in only $61 \%$ of the cases.

In our study, we saw an average static 2-point discrimination (2-PD) of $10.57 \mathrm{~mm}$.

Tränkle et al. [17] investigated the quality of sensibility from innervated FDMCA island flaps in younger and older patients and evaluated the donor site morbidity at the index finger. The mean age of the patients was 48.3 years, and the mean follow-up period was 3 years. The 14 patients older than 50 years had a static 2-point discrimination (s2PD) of $10.9 \mathrm{~mm}$ compared with $10.8 \mathrm{~mm}$ of the 11 patients younger than 50 years. The average loss of s2-PD of the flap compared with the donor area averaged $2.7 \mathrm{~mm}$ in all patients. Complete cortical reorientation occured in seven 
patients older than 50 years and in five patients younger than 50 years. Total loss of range of motion of all donor finger joints was $14^{\circ}(4.4 \%)$ compared with the contralateral index finger. Twenty-two patients were satisfied with the result. There were no significant age-related differences in the surgical results of the innerved FDMCA island flap, and donor site morbidity was negligible.

Shun-Cheng et al. [18] also described the sensate first dorsal metacarpal artery flap for resurfacing extensive pulp defects of the thumb. They reported eight patients with extensive pulp defects of the thumb over a period of 3 years. Skin defects in all patients were combined with bone, joint, or tendon exposure. All flaps survived completely. The eventual static 2-point discrimination of the flap ranged from 6 to $14 \mathrm{~mm}$, and the patients needed 4-8 months to reorient the flap in the new location. The skin grafts applied to the donor area were satisfactory, and full recovery of flexion and extension of the index finger was also obtained.

\section{Conclusion}

The FDMCA flap has a constant anatomy and easy dissection. It has a low donor site morbidity, if FTSG is used. It shows good functional and aesthetic results. The FDMCA flap is a first treatment of choice for defects of the proximal phalanx and proximal part of the distal phalanx of the thumb.

\section{References}

1. Hilgenfeldt O (1950) Operativer daumenersatz. Enkeverslag, Stuttgart

2. Holevich J (1963) A new method of restoring sensibility to the thumb. J Bone Joint Surg 45B:496-502
3. Foucher G, Braun JB (1979) A new island flap transfer from the dorsum of the index to teh thumb. Plast Reconstr Surg 63:344349

4. Gebhart B, Meissl G (1995) An extended first dorsal metacarpal artery neurovascular island flap. J Hand Surg 20B:529-531

5. Wilhelm K, Putz R, Hierner R et al (1997) Lappenplastiken in der Handchirurgie. Angewandte Anatomie, Operationstechniken, Differentialtherapie. Urban and Scharzenberg, München-WienBaltimore

6. Littler JW (1953) The neurovascular pedicle method of digital transposition for reconstruction of the thumb. Plast Reconstr Surg 12:303-319

7. Strauch B, Tsur H (1978) Restoration of sensation to the hand by a free neurovascular flap from the first web space of the foot. Plast Reconstr Surg 62:361-367

8. Buncke HJ, Rose EH (1979) Free toe-to-fingertip neurovascular flaps. Plast Reconstr Surg 63:607-612

9. Adani R, Cardon LJ, Castagnetti C, Pinelli M (1999) Distal thumb reconstruction using a mini wrap-around flap from the great toe. J Hand Surg 24B:437-442

10. Ratcliffe RJ, Regan PJ, Scerri GV (1992) First dorsal metacarpal artery flap cover for extensive pulp defects in the normal length thumb. Br J Plast Surg 45:544-546

11. Sherif MM (1994) First dorsal metacarpal artery flap in hand reconstruction. I. Anatomical study. J Hand Surg 19A:26-31

12. Sherif MM (1994) First dorsal metacarpal artery flap in hand reconstruction. II. Clinical application. J Hand Surg 19A:26-31

13. Eski M, Nisanci M, Sengezer M (2007) Correction of thumb deformities after burn: Versatility of first dorsal metacarpal artery flap. Burn 33(1):65-71

14. Pelissier P, Casoli V, Bakhach J, Martin D, Baudet J (1999) Reverse dorsal digital and metacarpal flaps: a review of 27 cases. Plast Reconstr Surg 103(1):159-165

15. El Khatib HA (1998) Clinical experiences with the extended first dorsal metacarpal artery island flap for thumb reconstruction. J Hand Surg [Am] 23(4):647-652

16. Yoshinori O (2000) Sensory function of the neurovascular island flap in thumb reconstruction: comparison of original and modified procedures. J Hand Surg [Am] 25A:637-643

17. Tränkle M, Sauerbier M, Heitmann C, Germann G (2003) Restoration of thumb sensibility with the innervated first dorsal metacarpal artery island flap. J Hand Surg [Am] 28(5):758-766

18. Shun-Cheng C, Shao-Liang C, Tim-Mo C, Chia-Jueng C, TianYeu C, Hsian-Jenn W (2004) Sensate first dorsal metacarpal artery flap for resurfacing extensive pulp defects of the thumb. Ann Plast Surg 53(5):449-454 